\title{
Choosing optimal rapid manufacturing process for thin-walled products using expert algorithm
}

\author{
Filip Górski, Wiesław Kuczko, Radosław Wichniarek, Adam Dudziak, \\ Maciej Kowalski, Przemysław Zawadzki \\ Poznan University of Technology (POLAND) \\ filip.gorski@,doctorate.put.poznan.pl;wieslaw.kuczko@,doctorate.put.poznan.pl; \\ radoslaw.wichniarek@doctorate.put.poznan.pl; adam.dudziak@put.poznan.pl; maciejkow@poczta.fm; \\ przemyslaw.zawadzki@put.poznan.pl
}

Received February 2010

Accepted October 2010

\begin{abstract}
Choosing right Rapid Prototyping technology is not easy, especially for companies inexperienced with that group of manufacturing techniques. Paper summarizes research focused on creating an algorithm for expert system, helping to choose optimal process and determine its parameters for thin-walled products rapid manufacturing. Research was based upon trial manufacturing of different thin-walled items using various RP technologies. Products were categorized, each category was defined by a set of requirements. Basing on research outcome, main algorithm has been created. Next step was developing detailed algorithms for optimizing particular methods. Implementation of these algorithms brings huge benefit for recipients, including cost reduction, supply time decrease and improvements in information flow.
\end{abstract}

Keywords: decision process, rapid manufacturing, optimization

\section{Introduction}

Rapid prototyping consists of various manufacturing processes by which a solid physical model of a part is made directly from 3D computer-aided design (CAD) model data. This CAD data may be generated by 3D CAD Modelers, CT and MRI 
scan data or model data created by 3D digitizing systems (Engineers Handbook, 2006). The Rapid Prototyping techniques have found their place among other manufacturing technologies. They are invaluable when there is a need of quick producing a physical prototype of designed part. In case of necessity of manufacturing a small series of functional products (especially those who would otherwise need dedicated special tooling) it is also hard to find a competitive group of technologies to accomplish the task. The same situation occurs when there is a need of creating a part with very complex geometrical features.

There is a plethora of different rapid prototyping and manufacturing technologies. Each one has its own set of applications, pros and cons and defined level of costs. It is, however, hard to judge which technique is the most appropriate for use in specific task, but this choice is very important in terms of achieving satisfying end results. Choosing the wrong method can have a negative impact on financial results of a company that requested to make a series of products using inappropriate technology.

Process of selecting the suitable technology and its parameters can be difficult, especially for companies not aware of all benefits (or threats) possible to obtain by application of rapid prototyping and manufacturing techniques. Moreover, information exchange between company ordering prototypes and company providing this kind of service can be in such case long and time-consuming, while the most important benefit gained with rapid prototyping is the possibility of saving a considerable amount of time. If the information exchange is too long, benefits can be diminished in a significant way. If the information flow is incomplete and inaccurate it can have distinct negative effects - produced parts will not fulfil the requirements. In consequence it will bring the financial losses on both sides, it can also have other negative effects hard to calculate (like losing an important client).

All these considerations come out of a long-term cooperation between Rapid Prototyping Laboratory on Poznan University of Technology and companies that make use of its services in rapid manufacturing and prototyping. A need of improving a process of selecting an appropriate technology and its parameters emerged - especially for thin-walled products, which are the most requested type of products. 
As a result of research that has been carried out to solve that problem, an expert algorithm of selecting the right rapid prototyping technique for thin-walled products has been created. The algorithm is a base for creating an expert system for technology selecting. An expert system is comprehended as a computer program that simulates the thought process of a human expert to solve complex decision problems in a specific domain (Badiru \& Cheung, 2002).

Application of the system in process of information exchange between client and manufacturer can bring a number of benefits, including shortening time spent on sending inquiries, cost optimization, delivery time reduction and improving product quality.

The algorithm has been created for technologies available in the Laboratory. There is however no limitation of expanding its range on other known and popular generative manufacturing technologies.

\section{Practical research}

\subsection{Characteristics of selected Rapid Prototyping methods}

Practical research was carried out in the Laboratory of Rapid Prototyping on Poznan University of Technology. There are three RP technologies available in the Laboratory.

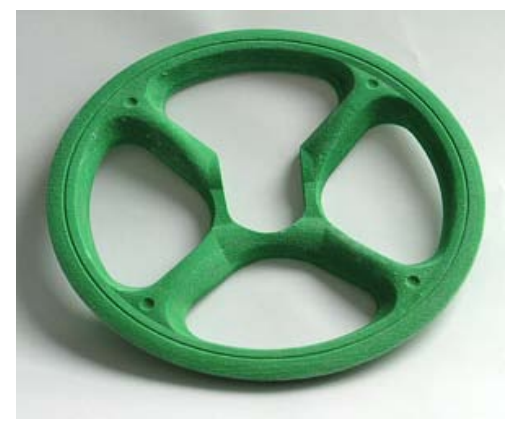

Figure 1. "Model made using 3D Printing technology".

3D Printing (3DP) - printing in three dimensions. Physical model is built by layering of powdered material, selectively bonded by the jetting of adhesive (Adams, 2008). Model can be hardened by soaking it with appropriate resin or cyanide acrylate, which ensures appropriate strength and functional properties. Main advantages of 
this technique is a short time of obtaining a part and low costs. Another virtue of this method is the availability of colored materials and the ability to create a multicolored part (Adams, 2008). Models can be finished with post processing, they can also be assembled with gluing. An example of model created with this technique is shown on Figure 1.

Fused Deposition Modeling (FDM) - here plastic filament supplied through a heated nozzle, where it becomes plastified, is used to build a model. Models are created by layering two types of material through two-nozzled head - one of them is the model material, the other one is the supporting material. Numerically controlled device puts on subsequent layers of ABS model material and supporting material, basing on 3D model. Resulting part is an accurate representation of the design and is ready for use instantly. There is a possibility of using different colors. The key advantage is a short time of getting a fully functional part without need of postprocessing. Produced models have considerable strength and durability and can be further processed by machining, gluing or painting, giving the surface a desired quality. Model made using this technique is shown on Figure 2.

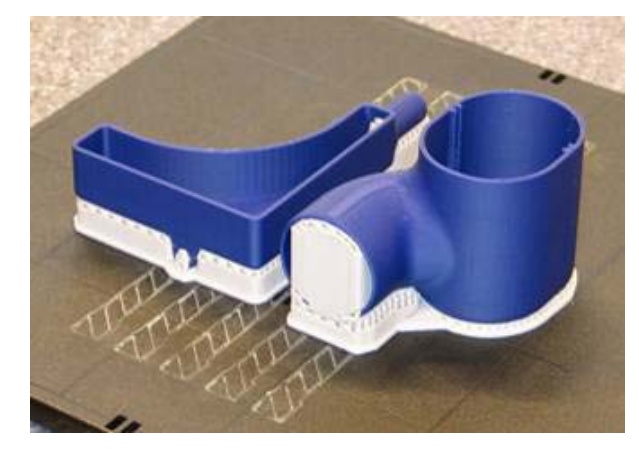

Figure 2. "Models made with FDM (grey support material can be seen)".

Vacuum Casting (VC) - casting using silicone mould, in conditions of vacuum. Elastic mould of silicone rubber is poured with two-component resin that imitates properties of various plastics (Figure 3 ). Depending on used resin, color and admixtures, castings with desired mechanical and visual properties can be acquired. Specific properties (like transparency or elasticity) are also available to obtain, after adding appropriate admixtures. Silicone mould can be also poured with wax, resulting model can be used to make a sand mould for metal castings. Also, there is a possibility of using silicone moulds to create metal parts - low melting temperature alloys must be used. Applying resins with proper admixtures 
creates possibility of obtaining fully functional tooling elements, like dies, stamps and injection mould inserts. Advantage of this method is a low cost of mould in comparison of traditional methods, great surface mapping and repeatability. Vacuum Casting is an excellent method for producing short series of durable and fully functional products.

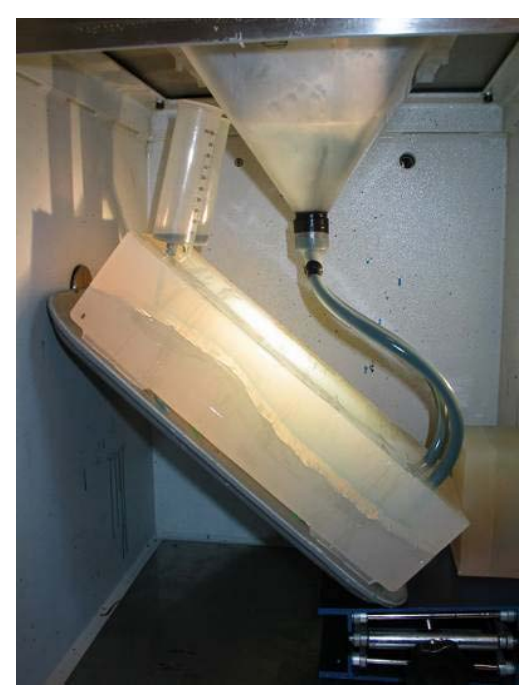

Figure 3. "Silicone mould, placed in vacuum chamber, after pouring with resin".

\subsection{Thin-walledness criterion}

Thin-walledness is a property of object resulting directly from its geometry. Product can be qualified as thin walled if material thickness (measured in direction normal to a plane tangent to object surface) in any place is significantly lower than other object dimensions. Thus, thin-walledness is strictly dependent on product overall dimensions - having the same thickness one of products can be qualified as thin-walled while the other one cannot. In case of research described in this paper natural limitation of overall dimension was size of the working chambers of machines used to carry out particular processes. Consequently, a criterion has been set that every object with thickness below 6 millimeters will be considered as thin-walled.

Exemplary models of thin-walled products has been presented on Figure 4. 

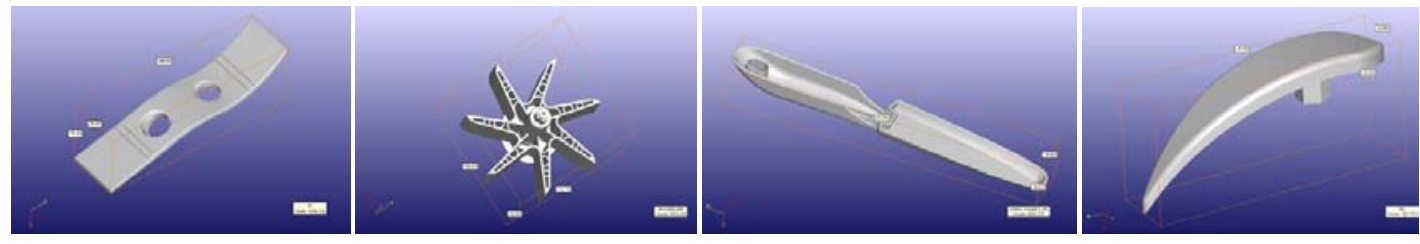

Figure 4. "Examples of thin-walled products".

\subsection{Prototype division criteria}

As a result of analysis of different rapid prototyping processes with available methods and considering application of created physical models the following criteria of division has been developed:

Group I - visual prototypes. Requirements concern only dimensions, surface quality and colour - these parameters have to be as close to design (or original part, if the prototype is based on existing part) as it is possible. Functionality is not required from these prototypes. Substantial limitation in case of thin-walled products belonging to this group is ensuring an appropriate surface quality. In most cases small elements and complex shapes of the model make any further processing very hard, but this processing is necessary to fulfil preliminary requirements.

Group II - functional prototypes (Figure 5), apart from dimensional compatibility some durability and strength along with functional properties are also required (like flexural strength, possibility of assembling with other elements). Some material properties (like humidity resistance) may also be required. Prototypes which do not need to resemble the design colourwise also belong to this group. In cases of prototypes from group II, post-processing mostly does not occur.

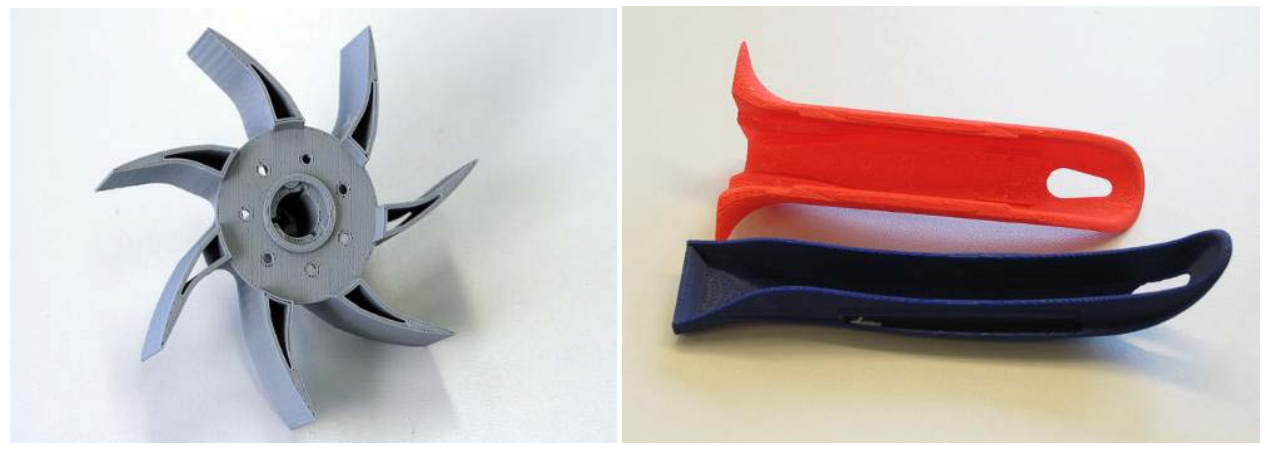

Figure 5. "Examples of thin-walled functional prototypes". 
Group III - fully functional prototypes (Figure 6), their surface quality and color has to resemble the design as most as possible, mechanical (flexural, tensile and impact strength, hardness) and material properties (incombustibility, humidity and atmospherical hazards resistance) has also to stay on an acceptable high level. Mostly in case of prototypes belonging to that group, an important factor is the possibility of obtaining a precise number of prototypes (small batches).

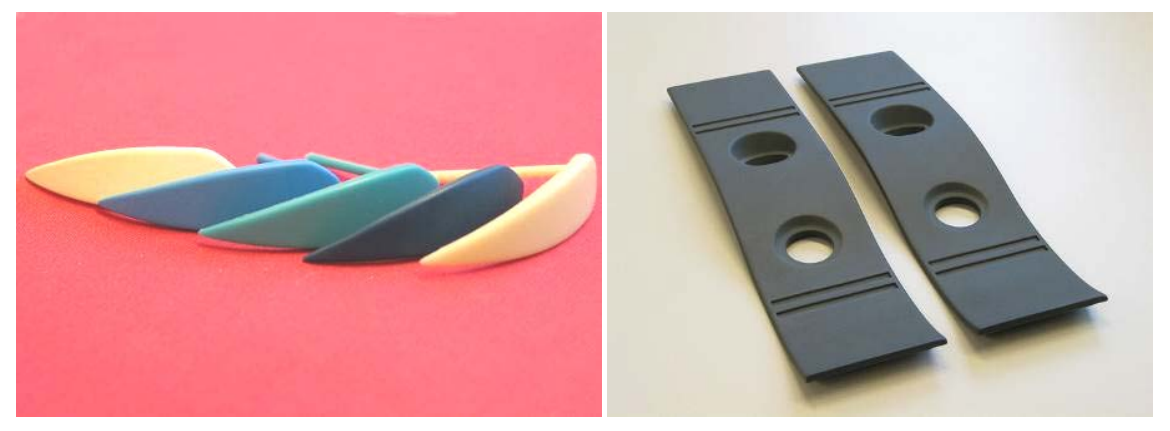

Figure 6. "Examples of thin-walled usable prototypes".

\subsection{Research course and its results}

During the research a number of thin-walled prototypes from different groups (as per presented division criteria) has been produced using available rapid prototyping technologies. Work was aimed at examining the usability of particular techniques for making different sorts of prototypes. Also, tests on optimization of particular processes concerning mostly surface quality, time and cost of manufacturing were undertaken. Furthermore, connections between ways of realizing the particular processes and mechanical properties of resulting prototypes were examined. The following conclusions have been made:

1) 3D Printing, despite low costs and the shortest time of creating the part, does not give the possibility of getting satisfying results when making prototypes from II and III group. Basic limitation in case of 3DP technique is model brittleness before final hardening. Application of appropriate hardening substances can solve this problem, but in case of thin-walled products it is very hard to perform and often leads to deformation or partial destruction of the model. 3D Printing can be successfully used for making prototypes from group I or base model, which is then used to create a silicone mould for Vacuum Casting process. 
2) FDM technique has many more possibilities than 3DP. Properties of the model material $(A B S)$ in most cases are good enough to make prototypes belonging to group I or II and also a base models for Vacuum Casting process. However, there are problems with visual quality of the models. When creating visual and fully functional prototypes, surface finishing is an important matter. Because of laminar structure of the model, visual quality is decreased by the so-called "step effect". Post-processing is necessary - it is carried out by a manual model processing (polishing, smoothing, painting, etc.). Although with FDM models having thickness of $0,6 \mathrm{~mm}$ are available to obtain, preparing the surface to meet the requirements is very time-consuming and unprofitable with higher number of pieces. Thus, FDM technique is the most suitable for making prototypes belonging to group II, in small numbers.

3) Prototypes of group III, as the most demanding, are manufactured using all available rapid prototyping techniques. For making short series of products with full functionality, Vacuum Casting is one of the most suitable technologies. After performing a proper analysis a conclusion can be made that achieving high mechanical properties of prototype does not mean that visual quality has to be poor (Górski \& Wichniarek, 2010). By selecting appropriate resins, colours and admixtures, fully functional ready plastic part, meeting all the requirements, can be obtained. During the research, tests on optimization of mould creating and casting processes have been undertaken, mostly aimed at shortening the time of making the finished product and its correctness. The research allowed to correlate the basic factors: quality, labour consumption and price of the end product.

\section{Algorithm of technology selection}

As a result of performed research, an algorithm of selecting the right rapid prototyping process and its detailed parameters has been created. In original form the algorithm is very complex and because of that, shorter and simplified version has been created. This simple version is more suitable for presentation and it shows the basic ideas behind the algorithm. It is shown on Figure 7.

Rapid prototyping techniques can be used in the process of developing a new product (as a prototyping method), as well as in reverse engineering processes, as a technology of re-creating an existing part. Hence the presented algorithm has 
several start fields - in case of a new product a 3D model obtained during the design process is the base, further actions are performed after its implementation. Existing product - if it does not need any modifications - can become a base itself, as a basic model for creating silicone mould for Vacuum Casting process. If existing part needs geometrical modifications, digitization process (obtaining a digital model with one of available methods) has to be performed. After obtaining the digital model, necessary modifications can be made. Then the algorithm of technology selection is continued the same way like with new product.

Depending on requirements on type, destination and desired properties of the product, there are different paths available leading to choice of optimal process and determining its parameters. The algorithm has been designed to comprise only the techniques available during the research. Hence all possible to obtain parameters, concerning the product (accuracy, surface quality, hardness, impact strength etc.) and the process (time, costs) cannot exceed the characteristic parameters of specific techniques. If, during the selection process using the algorithm, a parameter of magnitude impossible to achieve with available techniques will be put in, an information will be generated about the need of using entirely different technology. It is not shown directly in the presented algorithm, because there are too many places when such information could occur - presented algorithm would become illegible.

End result of performing the algorithm is the set of information. Part of them should be sent to client - delivery time, price and expected mechanical properties of products are among them. The remaining information are for the manufacturer they contain parameters of realized processes, surface quality requirements, material data, time requirements and other important information.

In present form, an algorithm is performed by the manufacturer, basing on information sent by client using electronic or direct means of communication. Creating an algorithm allowed to simplify the process of technology selecting for clients, which do not have an appropriate knowledge regarding available rapid prototyping technologies. 


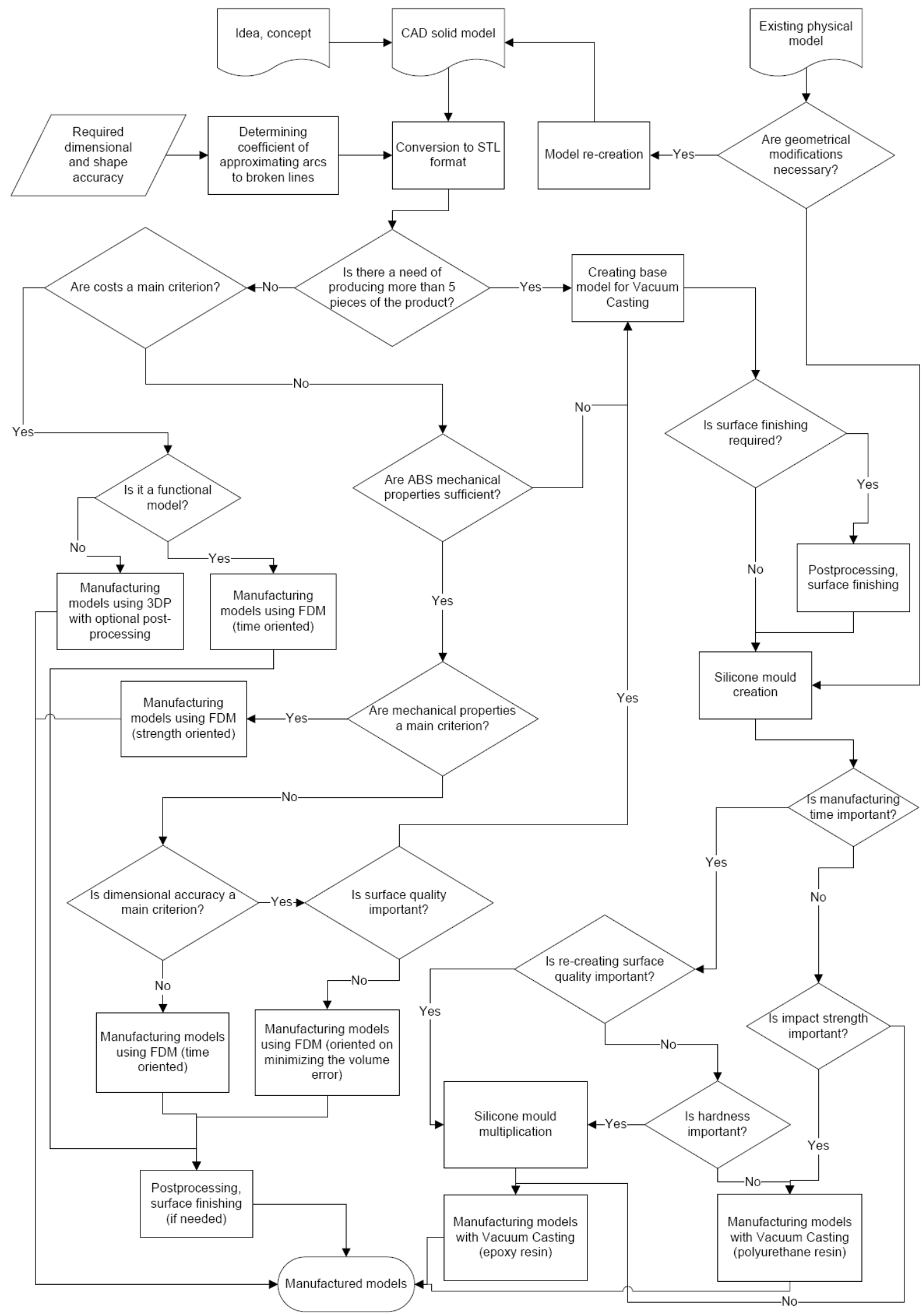

Figure 7. "Simplified form of algorithm for rapid prototyping technology selection". 


\section{Further research and development possibilities}

\subsection{Multi-criterial expert system}

As for now, created algorithm does not have a possibility of automatic realization. The process of selecting the technology and its parameters is carried out manually. Algorithm is also not available in any form to the client - he has to supply all the information to the manufacturer by the accessible means of communication, which can be arduous at times.

Creating the algorithm is only the first step on a way of optimizing the process of rapid prototyping technology selecting. The next step will be creation of multi-criterial expert system for selecting the appropriate rapid manufacturing and prototyping technology for thin-walled products in a form of computer program. The program will give the possibility of automatic technology and parameters selection, basing on input set of data. The information for both client and manufacturer will be also automatically generated by the system.

The program could be implemented in an on-line version, as a form or applet placed on internet website. Thanks to this, it could be easily accessible by the trustworthy clients. They could quickly check which technology will be the most appropriate for them, also receiving information about approximate time and costs of the process.

\subsection{I mplementation of other rapid prototyping technologies}

The expert system described in previous sub-chapter will be provided with the possibility of expanding it with new rapid manufacturing and prototyping technologies. Implementing a new process will be related with modifications of the base algorithm, to create a possibility of choosing that new technology as an optimal for fulfilling stated requirements.

Introducing other rapid prototyping technologies into expert system makes a number of practical examinations necessary, to compare them with available techniques in terms of meeting the clients requirements.

Ultimately, the expert system will be available to implement in every company using rapid prototyping technologies. Thus, system must be based on the data 
about all possible rapid prototyping technologies and during implementation of the system its range will be limited only to the technologies available in the specific company.

\subsection{I mplementation of other rapid prototyping technologies}

Final expert system should also contain the possibility of integration with existing management systems implemented in the specific company - especially with scheduling systems. Thanks to that integration, a preliminary planning of rapid prototyping workplaces load will become possible and information about delivery time, obtained by the client after ordering the products will become more real. The expert system, apart from selecting the technology and its parameters will give the possibility of generating information for the warehouse and purchase department. Thanks to this, integration of rapid prototyping technologies with company production system will become much easier.

\section{Algorithm of technology selection}

Created algorithm of selecting an optimal rapid prototyping technology for thin-walled products brings a number of benefits, both for manufacturer and the recipient of the final product. Optimization of applied techniques allows to choose a right process and its parameters in short time, in response for clients requirements. Thanks to this, great time savings are possible - it helps to maximize the benefits of rapid prototyping application. Optimization of product properties will give a possibility to give the client exactly what he wanted. It will help to avoid wasting clients money assigned for rapid prototyping.

Implementation of the algorithm will improve the information exchange process between manufacturer and recipient - both sides will get a necessary set of detailed information concerning ordered product, which will allow avoiding misunderstandings concerning delivery time and product quality.

Further development of the idea by creating a computer expert system will give the possibility to increase benefits of the companies which will implement it. Possibility of connecting the expert system with production management system already implemented in company will allow taking the full advantage of possibilities connected with using rapid prototyping and manufacturing technologies. 


\section{References}

Adams L. (2008). Materials play the part. applianceDESIGN, 10, 12-21.

Badiru A.B., \& Cheung J.Y. (2002). Fuzzy engineering expert systems with neural network applications. New York: John Wiley \& Sons, Inc.

Engineers Handbook (2006). Introduction to RP. Retrieved March $3^{\text {rd }}, 2010$ from http://www.engineershandbook.com.

Górski, F., \& Wichniarek, R. (2010). Application of photohardening materials in rapid prototyping. Journal of Mechanical Engineering and Production Management, $13,25-33$.

(c) Journal of Industrial Engineering and Management, 2010 (www.jiem.org)

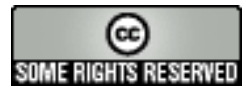

Article's contents are provided on a Attribution-Non Commercial 3.0 Creative commons license. Readers are allowed to copy, distribute and communicate article's contents, provided the author's and Journal of Industrial Engineering and Management's names are included. It must not be used for commercial purposes. To see the complete license contents, please visit http://creativecommons.org/licenses/by-nc/3.0/. 